\title{
Short-Term Forecasting of Temperature Driven Electricity Load Using Time Series and Neural Network Model
}

\author{
Nengbao Liu, Vahan Babushkin, and Afshin Afshari
}

\begin{abstract}
In this paper, two models were proposed for week-ahead forecasting of temperature driven electricity load, which are a time series model and an Artificial Neural Network (ANN) model. Over the week-long ("future") forecasting horizon, predicted temperature from ANN was used as it is shown that ANN produced more accurate temperature prediction. For the time series model, Seasonal Autoregressive Integrated Moving Average with eXogenous variables (SARIMAX) scheme was proposed. A method called "pre-whitening" was used to determine the lagged effect of temperature on electricity load. Comparison between ANN model and SARIMAX model was conducted to see which one gave a better forecasting performance. The forecast performance was characterized by two statistical estimates, the Root Mean Square Error (RMSE), Mean Absolute Percentage Error (MAPE). The results showed that while the ANN model behaved better in the estimation stage, its performance got worse than SARIMAX model in the forecasting stage.
\end{abstract}

Index Terms-Artificial neural networks (ANN), load forecasting, SARIMAX, short-term, temperature forecasting, time series.

\section{INTRODUCTION}

After the deregulation of electricity markets, electricity was commoditized. As a result, the generation of electricity more flexible and demand oriented. However, there are also risks associated the deregulation of electricity markets such as electricity oversupply and shortage due to inaccurate forecasting, which could result in significant financial loss. That is why accurate electricity forecasting plays a very important role and could also improve power generation planning. In this study two kinds of models, SARIMAX and ANN, were proposed for short-term forecasting of temperature driven electricity load forecasting.

Different approaches have been proposed for the short-term forecasting of electricity load. Generally speaking, these approaches can be grouped into three categories: regression-based, time series, artificial intelligence and computational intelligence. The latter can divided into several sub-groups, such as neural networks, support vector machines, hybrid and other approaches. In the following section, mainly neural networks and time series approaches will be studied from the literature.

Ghanbari et al. [1] and Elias et al. [2] both conducted a

Manuscript received September 29, 2013; revised December 20, 2013.

Nengbao Liu and Afshin Afshari are with the Department of Engineering System and Management Program, Masdar Institute of Science and Technology, Abu Dhabi, UAE. (e-mail: nliu@masdar.ac.ae, aafshin@masdar.ac.ae).

Vahan Babushkin is with the Department of Computing and Inforamtion Science, Masdar Institute of Science and Technology, Abu Dhabi, UAE. (e-mail: vbabushkin@masdar.ac.ae). comparison of two categories of models, ANN and Linear Regression. Whereas in Elias et al.'s paper [2], temperature was used to construct the models, which can also be found in Yong et al.'s [3], Neupane et al.'s [4] and Jennifer et al. [5] work. In addition to temperature, Beccali et al. [6] and Friedrich [7] included other weather variables, such as relative humidity and global solar radiation.

Paras et al. [8] Senabre et al. [9] and Martínez-Álvarez [10] all proposed an approach based on selection of similar days according to which the load curves are forecasted by using the information of the days being similar to that of the forecast day.

Choi et al. [11] and Kutluk et al. [12] both proposed the classic SARIMA method for load forecasting while James Taylor extended double seasonal ARMA model which includes intraday and intraweek seasonal cycles to include intrayear seasonal cycle, which is also apparent if one disposes of a multi-year training dataset. Weather features were also used to construct a classic ARMA/SARIMA model, which can be found in Jennifer et al.'s work. [5]

G. Peter [13] proposed a hybrid methodology that combines both ARIMA and ANN models to take advantage of the unique strength of ARIMA and ANN models in linear and nonlinear modeling.

The rest of the paper is organized as follows. In Section II, a brief description of the dataset used in this paper is presented. In Section III, a detailed structure of the ANN and SARIMAX models is described. In Section IV, the results of these two models are compared to evaluate their individual performance during both estimation and forecasting period. In Section V, the conclusion is reached and also future potential work is proposed.

\section{DATA DESCRIPTION}

The dataset used in this paper is mainly electricity load and dry bulb temperature from Abu Dhabi, UAE, which is under high temperature throughout the year. In this section, a general description of the dataset is given and we can see some characteristics of the dataset.

\section{A. Electricity Load}

The electricity load data used in this study is hourly load data for whole year of 2010 and the first half year of 2011 in mainland of Abu Dhabi, UAE. Fig. 1 shows the plot of load, where daily, weekly, annual seasonality, and trend is clearly visible.

Estimation period: whole year of 2010 and the first half year of 2011 except for the last one week for forecasting.

Forecasting period: last one week at the end of the first half year of 2011. 


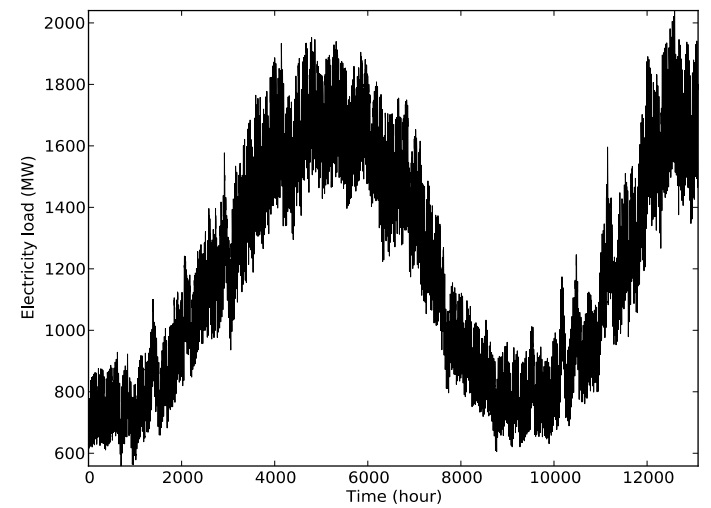

Fig. 1. Electricity load for year 2010 and the first half year of 2011.

\section{B. Temperature}

The temperature data used in this study is hourly dry bulb temperature for the whole year 2010 and the first half year of 2011 for Abu Dhabi, UAE. The Fig. 2 below shows the plot of temperature. It has a typical daily seasonality and a typical annual seasonality. However, there is supposed to be no trend and even if we consider the global warming, the effect would be negligible in the short term.

Estimation period: whole year of 2010 and the first half year of 2011 except for the last one week for forecasting.

Forecasting period: last one week at the end of the first half year of 2011 .

In the temperature forecasting, two models were proposed, ANN and SARIMA model respectively. The forecasted temperature used in this paper was from the ANN model which gave a better forecasting performance. In the forecasting, SARIMA gave an MAPE of $9.49 \%$ and a RMSE of $4.08^{\circ} \mathrm{C}$ whereas ANN gave an MAPE of $3.55 \%$ and a RMSE of $1.44{ }^{\circ} \mathrm{C}$.

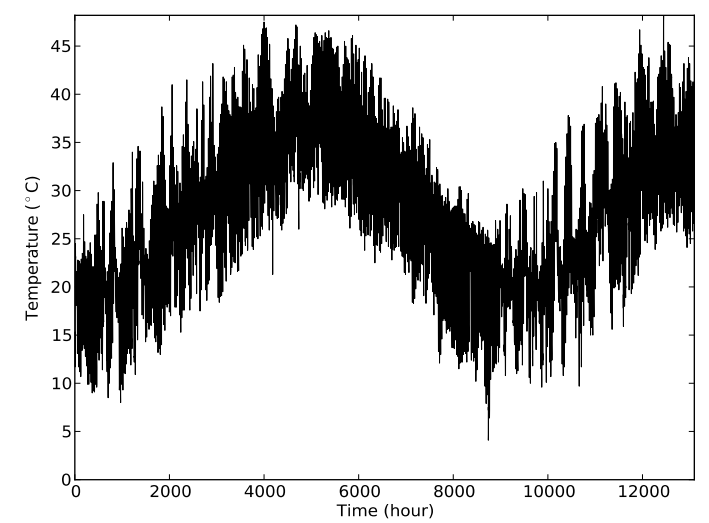

Fig. 2. Temperature for year 2010 and the first half year of 2011.

\section{Methodology}

The models proposed in this paper are ANN and SARIMAX respectively. In this section, we will see a detailed structure of both models.

\section{A. ANN}

\section{1) Input data}

In this study, three types of input variables are used:

- Load data:

Load data is notoriously autocorrelated at multiple lags. In considering this, previous 1 to 6 hours load, previous 12 hours load, previous day same hour load, previous two days same hour load were chosen as input in order to model the autocorrelation.

- Dummy variables:

Hourly dummy variables characterize hour of day, day of week, month of year, Ramadan and holidays.

- Exogenous variable:

In this study, one exogenous variable, dry bulb temperature was included. In regions with extreme climate, such as the UAE, load is highly correlated with load. Given the fact that temperature has lagged effect on load (due, e.g., to the thermal inertia of buildings), in addition to current temperature, lagged values at lags $1,2,6,12$, and 24 were used.

\section{2) Model structure}

- Hidden layer size

Our literature review reveals that rarely more than one hidden layer was used. So in this study just one hidden layer was adopted. For the number of neurons in hidden layer, there is no universal rule, so the only way would be that one should check the performance of different models using different number of neurons in the hidden layer while at the same time being wary of over-fitting issue. After several trials, one hidden layer with 10 neurons was chosen as it performed quite well.

- Data division

In Neural Network Toolbox of MATLAB, the embedded function divides the data into three parts: training part, validation part and test part. Training part plus the validation part corresponds to estimation period while the rest test part corresponds to forecasting period. In dividing the data of estimation period into training and validation part: ten thousands data points constituted the training part and the remaining data (2755 data points) constituted the validation part. The data of forecasting period was a week long (168 data points).

- Algorithm

The default training algorithm in the Neural Network Toolbox of MATALB is the Levenberg-Marquardt algorithm, which was also the algorithm used in this study.

\section{B. SARIMAX}

\section{1) Data pre-processing}

- Threshold function

For temperature, a threshold function was applied to account for the nonlinear relationship between temperature and load. The cut-off point identified in this study was $18.5^{\circ} \mathrm{C}$, at which electricity starts being used for indoor space cooling. When temperature is lower than the cut-off point, it is set to zero, while temperature is higher than the cut-off point, its value is the subtraction of the original value and cut-off value.

- Removing non-stationarity

As the load has three types of seasonality, which are daily, weekly and annual seasonality, three differencing at lag 24, 168 and 8760 were applied respectively.

Also, since the load has a trend, a first order differencing was applied besides the differencing at seasonal lags. After the application of those four differencing operators, as shown by the rapidly decaying autocorrelation (ACF) and partial autocorrelation (PACF) plots in Fig. 3, the time series seems to be almost stationary. 

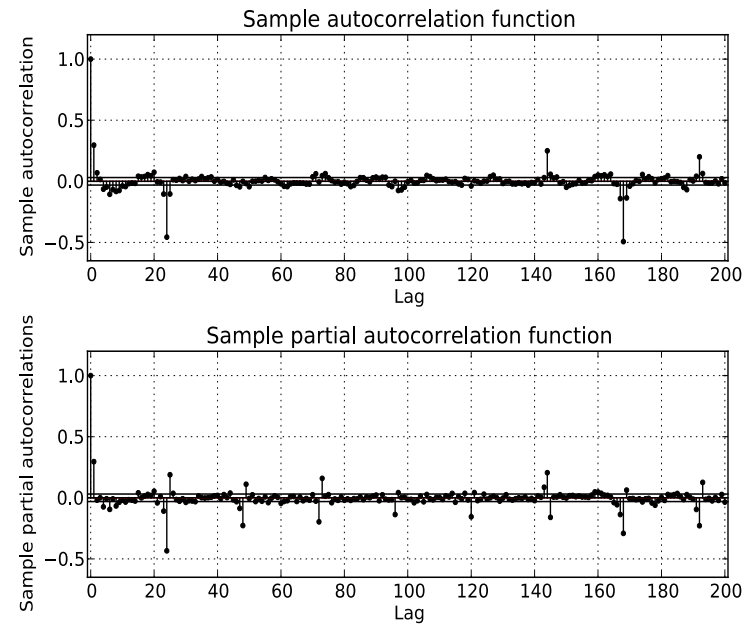

Fig. 3. ACF and PACF for first order, daily, weekly and yearly differenced load.

\section{2) Full model description}

The model to be identified is expressed as equation below shows.

$$
\begin{gathered}
y_{t}=\frac{\omega(B)}{\delta(B)} x_{t}+\frac{\theta(B)}{\phi(B)} a_{t} \\
y_{t}=(1-B)\left(1-B^{24}\right)\left(1-B^{168}\right)\left(1-B^{8760}\right) Y_{t} \\
x_{t}=(1-B)\left(1-B^{24}\right)\left(1-B^{8760}\right) X_{t}
\end{gathered}
$$

where $Y_{t}$ is original load data, $y_{t}$ is the stationarized load data, $X_{t}$ is original temperature data, $x_{t}$ is the stationarized temperature data and $a_{t}$ is the innovation. $\frac{\omega(B)}{\delta(B)}$ is the transfer function to be identified to model lagged effect of temperature on load. $\phi(B)$ is AR and SAR polynomial and $\theta(B)$ is MA and SMA polynomial for load.

\section{3) Pre-whitening}

As temperature has lagged effect on load, a transfer function for temperature was to be identified to model its lagged effect on load. A common way is to apply a transformation on both temperature and load. The transformation is to make temperature a white noise, which is called pre-whitening and then the same transformation is applied on load. Fig. 4 shows the pre-whitened temperature, which ideally is a white noise.
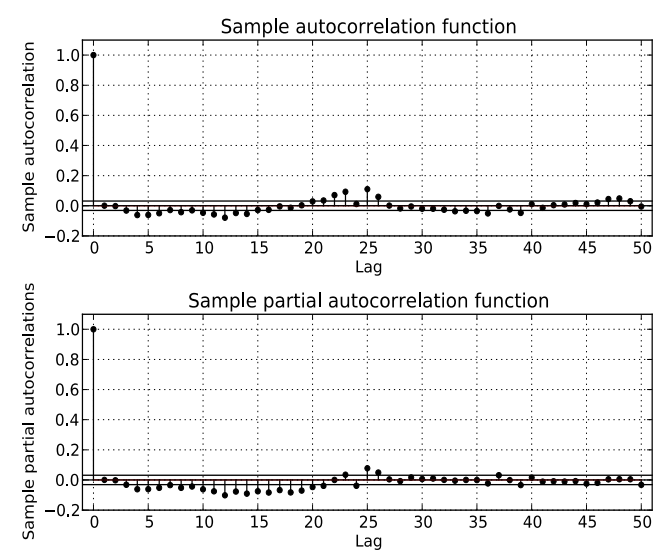

Fig. 4. ACF and PACF for pre-whitened temperature.

\section{4) Cross-correlation}

After we applied transformation for both temperature and load, we calculated the cross-correlation between those two transformed time series at different lags and checked the plot of cross-correlation, as Fig. 5 shows. Comparing it with those plots from Wei [14], a transfer function of form was specified.

$$
v(B) x_{t}=\frac{\omega_{0}}{1-\delta_{1} B} x_{t}
$$

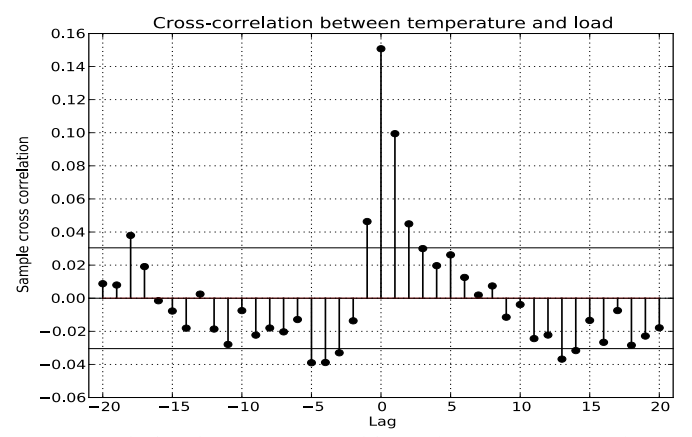

Fig. 5. Cross correlation between pre-whitened temperature and transformed load.
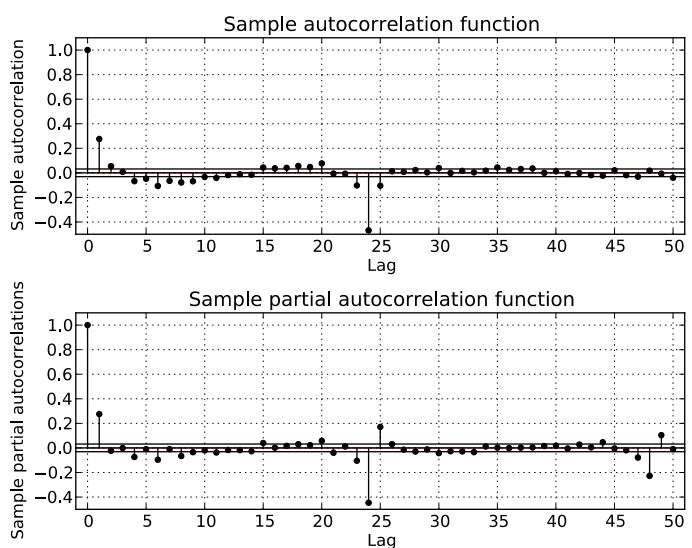

Fig. 6. ACF and PACF for $n_{t}$

\section{5) SARMA model specification for $n_{t}$}

After we identified the transfer function $v(B) x_{t}=$ $\frac{\omega_{0}}{1-\delta_{1} B} x_{t}$, we obtained $n_{t}$ as equation below shows,

$$
n_{t}=y_{t}-\hat{v}(B) x_{t}
$$

Fig. 6 shows the ACF and PACF of $n_{t}$, we can see that there is an obvious autocorrelation and partial autocorrelation at lag 1 and 24. After checking ACF and PACF, an SARMA model was chosen to model $n_{t}$.

For the order of autoregressive and moving average in the seasonal component, as there is obvious autocorrelation at lag 24, we included a SAR and SMA part with order one at lag 24, which were supposed to model the correlation at lag 24 .

For the order of autoregressive and moving average in the non-seasonal component, we identified the best value through Akaike Information Criterion (AIC).

From the AIC analysis, the optimal value of 5 was identified for the order of autoregressive and moving average in the non-seasonal component. For simplicity and also given the ACF and PACF of $n_{t}$, a SARMA $(1,1) \times(1,1)_{24}$ was chosen to model it.

Equation below shows the model of SARMA identified.

$$
\begin{aligned}
\left(1+\phi_{1} B\right)\left(1+\phi_{24} B^{24}\right) & n_{t}= \\
& \left(1+\theta_{1} B\right)\left(1+\theta_{24} B^{24}\right) a_{t}
\end{aligned}
$$

\section{6) Full model estimation}

After having fully specified the different components of the model, all the parameters of the model were identified simultaneously. The full model is showed in equation. 


$$
\begin{gathered}
y_{t}=\frac{\omega_{0}}{1-\delta_{1} B} x_{t}+\frac{\left(1+\theta_{1} B\right)\left(1+\theta_{24} B^{24}\right)}{\left(1+\phi_{1} B\right)\left(1+\phi_{24} B^{24}\right)} a_{t} \\
y_{t}=(1-B)\left(1-B^{24}\right)\left(1-B^{168}\right)\left(1-B^{8760}\right) Y_{t} \\
x_{t}=(1-B)\left(1-B^{24}\right)\left(1-B^{8760}\right) X_{t}
\end{gathered}
$$

where $\frac{\omega_{0}}{1-\delta_{1} B}$ is the transfer function to model temperature effect on load. $\left(1+\phi_{1} B\right)\left(1+\phi_{24} B^{24}\right)$ is AR and SAR polynomial and $\left(1+\theta_{1} B\right)\left(1+\theta_{24} B^{24}\right)$ is MA and SMA polynomial for load.

\section{RESUlTS AND ANALYSIS}

In this section, the results obtained using ANN and SARIMAX model are evaluated to compare the performance of each model.

\section{A. Performance Measurement}

RMSE and MAPE are frequently used measure of the differences between values estimated and predicted by a model or an estimator and the values actually observed. RMSE and MAPE are defined as equation shows.

$$
\begin{gathered}
R M S E=\sqrt{\frac{\sum_{t=1}^{n}\left(y_{t}-\hat{y}_{t}\right)^{2}}{n}} \\
M A P E=\frac{1}{n} \sum_{t=1}^{n}\left|\frac{y_{t}-\hat{y}_{t}}{y_{t}}\right|
\end{gathered}
$$

where $y_{t}$ is the actual value and $\hat{y}_{t}$ is the estimated or forecasted value.

\section{B. $A N N$}

\section{1) Estimation performance}

In the estimation period, an Mean Absolute Percentage Error (MAPE) of $0.47 \%$ and a Root Mean Square Error (RMSE) of 8.32 MW was obtained.

\section{2) Forecasting performance}

In the forecasting period, an MAPE of $3.57 \%$ and a RMSE of $72.92 \mathrm{MW}$ were obtained in forecasting. Fig. 7 shows the forecasted load verse the actual load in the forecasting period.

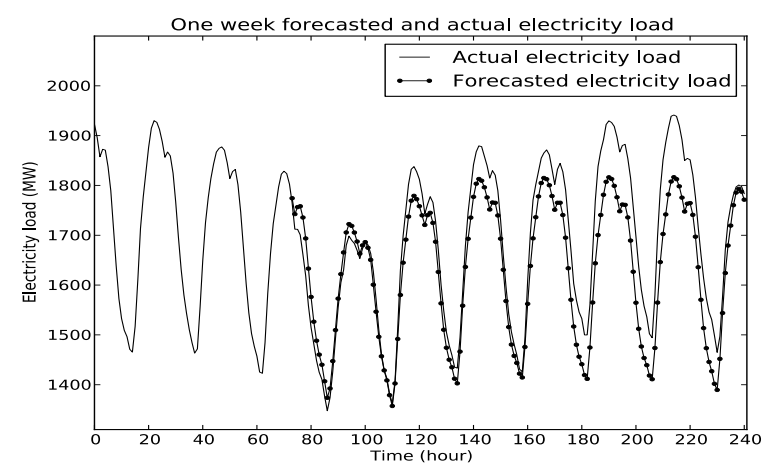

Fig. 7. Forecasted load and actual load in forecasting period using ANN model

\section{SARIMAX}

\section{1) Estimation performance}

In the estimation period, an MAPE of $0.98 \%$ and a RMSE of $15.80 \mathrm{MW}$ were obtained, which indicates a quite good fit to the estimation data but not as good as the MAPE of $0.47 \%$ and RMSE of $8.32 \mathrm{MW}$ with the ANN model.

\section{2) Forecasting performance}

In the forecasting period, an MAPE of $2.98 \%$ and a RMSE of 62.61 MW were obtained in forecasting, which is better than the MAPE of $3.57 \%$ and RMSE of $72.92 \mathrm{MW}$ obtained in ANN model. Fig. 8 shows the forecasted load verse the actual load in the forecasting period.

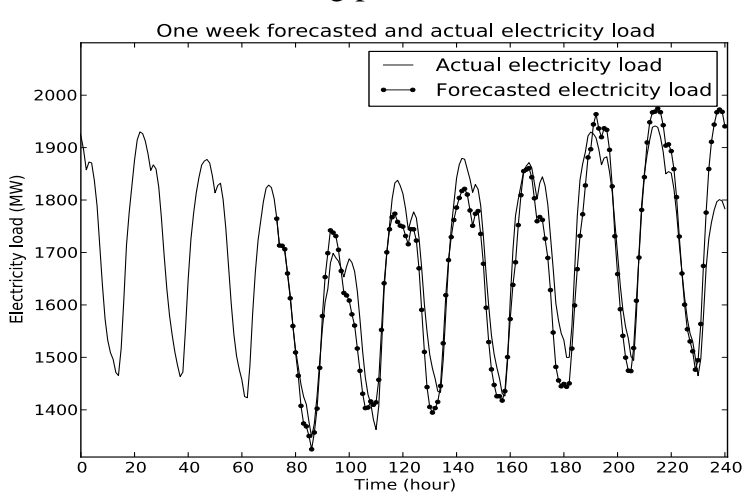

Fig. 8. Forecasted load and actual load in forecasting period using SARIMAX model

\section{CONCLUSION}

In this study, two models were proposed to forecast temperature driven electricity load, which are SARIMAX and Neural Network. Also one exogenous variable, temperature was included in both models. In order to forecast electricity load, temperature needs to be forecasted beforehand, which was accomplished with Neural Network given the result that an SARIMA model failed to give a more accurate forecasting for temperature. The "pre-whitening" method was used to determine the lagged effect of temperature on electricity load when constructing SARIMAX model. As Table I and Table II shows, although Neural Network achieved both lower RMSE and MAPE in estimation period, it failed to provide a better forecasting in a week forecasting period. This result would be explained by the fact that the SARIMAX model structure is more in agreement with the physical nature of the process as it explicitly incorporates the physical driving force of temperature on load, whereas ANN model just relies on the weights adjusted to achieve good fit. Future work would be to include adding more exogenous variables into the SARIMAX model (e.g., humidity and solar radiation). This would further enhance the forecasting performance of SARIMAX model.

TABLE I: ONE-WEEK FORECASTING RESULTS COMPARISON

\begin{tabular}{lllll}
\hline \hline Model & \multicolumn{2}{c}{ SARIMAX } & \multicolumn{2}{c}{ ANN } \\
\hline Period & Estimation & Forecasting & Estimation & Forecasting \\
RMSE & $15.80 \mathrm{MW}$ & $62.61 \mathrm{MW}$ & $8.32 \mathrm{MW}$ & $72.92 \mathrm{MW}$ \\
MAPE & $0.98 \%$ & $2.98 \%$ & $0.47 \%$ & $3.57 \%$ \\
\hline \hline
\end{tabular}

TABLE II: DIFFERENT TIME STEP FORECASTING RESULTS COMPARISON

\begin{tabular}{lllll}
\hline \hline $\begin{array}{l}\text { Forecasting } \\
\text { Period }\end{array}$ & \multicolumn{2}{c}{ SARIMAX } & \multicolumn{2}{c}{ ANN } \\
\hline & RMSE & MAPE & RMSE & MAPE \\
One day & $31.62 \mathrm{MW}$ & $1.58 \%$ & $40.53 \mathrm{MW}$ & $2.29 \%$ \\
One week & $62.61 \mathrm{MW}$ & $2.98 \%$ & $72.92 \mathrm{MW}$ & $3.57 \%$ \\
\hline \hline
\end{tabular}

\section{REFERENCES}

[1] A. Ghanbari, A. Naghavi, S. F. Ghaderi, and M. Sabaghian, "Artificial neural networks and regression approaches comparison for forecasting Iran's annual electricity load," in Proc. International Conference on Power Engineering, Energy and Electrical Drives, IEEE, 2009, pp. 675-679. 
[2] R. S. Elias, Liping Fang, and M. I. M. Wahab, "Electricity load forecasting based on weather variables and seasonalities: a neural network approach," presented at 8th International Conference on Service Systems and Service Management, June 25-27, 2011.

[3] Y. Wang, D. W. Gu, J. P. Xu, and J. Li. "Back propagation neural network for short-term electricity load forecasting with weather features," in Proc. International Conference on Computational Intelligence and Natural Computing, 2009, vol. 1, pp. 58-61.

[4] B. J. Neupane, K. S. Perera, Z. Aung, and W. L. Woon, "Artificial neural network-based electricity price forecasting for smart grid deployment," in Proc. International Conference on Computer Systems and Industrial Informatics, IEEE, 2012, pp. 1-6.

[5] J. Hinman and E. Hickey, "Modeling and forecasting short-term electricity load using regression analysis," Journal of Institute for Regulatory Policy Studies, 2009.

[6] M. Beccali, M. Cellura, V. L. Brano, and A. Marvuglia, "Forecasting daily urban electric load profiles using artificial neural networks," Energy Conversion and Management, vol. 45, no. 18, pp. 2879-2900, 2004.

[7] L. A. Friedrich, "An urban energy baseline model for measurement \& verification of building energy efficiency retrofits in Abu-Dhabi," M.S. thesis, Dept. Engineering System and Management Program, Masdar Institute, Abu Dhabi, UAE, 2013.

[8] P. Mandal, T. Senjyu, K. Uezato, and T. Funabashi, "Several-hours-ahead electricity price and load forecasting using neural networks," In Proc. Power Engineering Society General Meeting, IEEE, 2005, pp. 2146-2153.

[9] C. Senabre, S. Valero, and J. Aparicio, "Using a self organizing map neural network for short-term load forecasting, analysis of different input data patterns," Distributed Computing and Artificial Intelligence, Advances in Intelligent and Soft Computing, vol. 79, pp. 397-400, 2010.

[10] F. Martínez-Álvarez, A. Troncoso, J. C. Riquelme, and J. S. Aguilar-Ruiz, "LBF: A labeled-based forecasting algorithm and its application to electricity price time series," In Proc. Eighth IEEE International Conference on Data Mining, 2008, pp. 453-461.

[11] J. W. Taylor, "Triple seasonal methods for short-term electricity demand forecasting," European Journal of Operational Research, vol. 204, no. 1, pp. 139-152, 2010.

[12] T. M. Choi, Y. Yu, and K. F. Au, "A hybrid SARIMA wavelet transform method for sales forecasting," Decision Support Systems, vol. 51, no. 1, pp. 130-140, 2011.

[13] G. P. Zhang, "Time series forecasting using a hybrid ARIMA and neural network model," Neurocomputing, vol. 50, pp. 159-175.

[14] W. W. S. Wei, Time Series Analysis. Redwood City, California: Addison-Wesley, 1994. ch. 14, pp. 325.

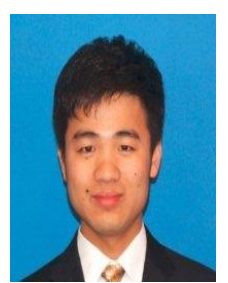

Nengbao Liu is currently a master student in Department of Engineering Systems and Management of Masdar Institute of Science and Technology, Abu Dhabi, UAE. His research focuses on modeling, analyzing and optimizing large-scale urban energy systems, assessing different demand side management measures towards efficient energy and infrastructure usage and decision support tools for policy making. 